\title{
Gastos públicos em serviços ambulatoriais de Fonoaudiologia no Brasil entre 2009 e 2018: bases de dados do DATASUS
}

\section{Public spending on outpatient speech therapy services in Brazil between 2009 and 2018: DATASUS databases}

\author{
Paula Carine Santos ${ }^{1} \mathbb{D}$, Caio Leônidas Oliveira de Andrade ${ }^{1} \mathbb{D}$, Elen Pereira de Jesus ${ }^{2} \mathbb{D}$,

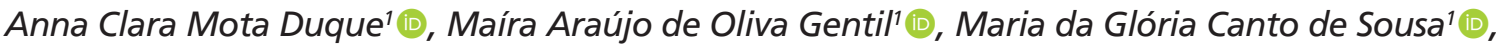 \\ Carla Cardoso ${ }^{10}$, Renata de Assis Fonseca Santos Brandão ${ }^{10}$, Gabriela Carvalho Machado ${ }^{2}$ (1)
}

\begin{abstract}
RESUMO
Objetivo: Analisar os gastos públicos em saúde destinados aos serviços ambulatoriais em Fonoaudiologia nas cinco Regiões Federativas do Brasil. Métodos: Trata-se de um estudo ecológico realizado por meio da busca de dados secundários disponíveis em uma plataforma virtual de domínio público, DATASUS (Departamento de Informática do Sistema Único de Saúde). Os dados coletados referiram-se aos valores aprovados para os procedimentos da Fonoaudiologia no período de 2009 a 2018 nas cinco Regiões Federativas. Adotou-se análise descritiva dos dados e exposição dos resultados em valores absolutos, relativos e taxas de crescimento. Resultados: Verificou-se que o investimento médio em reais $(\mathrm{R} \$)$, por ano, nos serviços de Fonoaudiologia no Brasil foi de, aproximadamente, R\$ 223.952.639.232,00; sendo 47,2\% dos recursos destinados à Região Sudeste. Dentre as grandes áreas de especialização, a Audiologia foi responsável por $95,4 \%$ do investimento, seguida pelas áreas da Linguagem $(4,0 \%)$, Motricidade Orofacial $(0,5 \%)$ e Voz $(0,1 \%)$. Conclusão: Os serviços fonoaudiológicos demandam considerável parcela dos recursos públicos, sendo a área da Audiologia responsável por quase metade desses gastos, seguida pelas grandes áreas da Linguagem, Motricidade Orofacial e Voz.
\end{abstract}

Palavras-chave: Sistema Único de Saúde; Fonoaudiologia; Pesquisa sobre Serviços de Saúde; Qualidade da Assistência à Saúde; Assistência à Saúde

\begin{abstract}
Purpose: To analyze public spending on health services to outpatient services in Speech Therapy in the five federative regions of Brazil. Methods: This is an ecological study carried out through the search for secondary data available on a public domain virtual platform, DATASUS. The collected data refer to the values approved for the Speech Therapy procedures in the period from 2009 to 2018 in the five federative regions. Descriptive analysis of data and exposure of results in absolute and relative values and growth rates were adopted. Results: It was found that the average investment in reais, per year, in speech therapy services in Brazil was approximately RS $223,952,639,232.00$, with $47.2 \%$ of the resources destined for the Southeast region. Among the major areas of specialization, Audiology accounted for $95.4 \%$ of the investment, followed by Language ( $4.0 \%)$, Orofacial Motricity $(0.5 \%)$ and Voice $(0.1 \%)$. Conclusion: The findings indicate that speech therapy services demand a considerable portion of public resources, with the area of Audiology being responsible for almost half of these expenses, followed by the large area of Language, Orofacial Motricity and Voice.
\end{abstract}

Keywords: Unified Health System; Speech Therapy; Health Services Research; Quality of Health Care; Delivery of Health Care

\footnotetext{
Trabalho realizado na Universidade do Estado da Bahia - UNEB - Salvador (BA), Brasil.

${ }^{1}$ Departamento de Ciências da Vida, Universidade do Estado da Bahia - UNEB - Salvador (BA), Brasil.

${ }^{2}$ Programa de Pós-Graduação em Processos Interativos dos Órgãos e Sistemas, Instituto de Ciências da Saúde, Universidade Federal da Bahia - UFBA - Salvador (BA), Brasil.

Conflito de interesses: Não.

Contribuição dos autores: CLOA e PCS foram responsáveis pela elaboração da ideia inicial da pesquisa e participaram da escrita do texto e da análise dos dados; EPJ, ACMD, MAOG, MGCS, CC, RAFSB e GCM participaram da escrita do texto, realizaram a normatização e as correções finais do artigo.

Financiamento: Nada a declarar.

Autor correspondente: Caio Leônidas Oliveira de Andrade. E-mail: caioleonidas@gmail.com

Recebido: Março 04, 2021; Aceito: Outubro 05, 2021
} 


\section{INTRODUÇÃO}

Nos últimos anos, os serviços de Fonoaudiologia no Sistema Único de Saúde (SUS) têm sido requisitados de forma crescente para oferecer uma prestação de serviço de qualidade, conforme preceitos da saúde pública. Em decorrência, muitos conceitos e práticas na Fonoaudiologia foram reavaliados ${ }^{(1)}$. Porém, ainda há muito que se fazer para que os princípios básicos do SUS sejam atingidos na sua totalidade, haja vista que o aumento da demanda de serviços fonoaudiológicos vem sendo alinhado à necessidade de crescentes investimentos e custos com uso de recursos públicos. Nessa perspectiva, a implantação de uma ferramenta sistemática de avaliação e controle dos serviços públicos de saúde favorece o aprimoramento da gestão dos recursos e da qualidade dos serviços ${ }^{(2)}$.

Assim, a discussão referente ao investimento em serviços de saúde deve ocorrer não apenas com base no montante de recursos destinados ao setor, mas na busca de técnicas e instrumentos que sejam eficientes e eficazes e contribuam para o acesso da população a um sistema de saúde público de qualidade, seguro e com bom custo-efetividade, no intuito de atingir os princípios básicos e as diretrizes do $\mathrm{SUS}^{(3)}$.

Ademais, notam-se, também, poucos estudos na Fonoaudiologia assentados em sistemas de informação e, ainda, pode-se afirmar que são escassos estudos que analisam os gastos públicos com procedimentos fonoaudiólogos, ou que analisam técnicas de auditoria, somados à inexistência de protocolos específicos em auditoria na área da Fonoaudiologia no Sistema Nacional de Auditoria (SNA) para sistematizar a prática, como acontecem de forma bem sólida em outras áreas da saúde, o que pode comprometer a qualidade assistencial para esses serviços ofertados pela rede SUS.

Face ao exposto, o presente estudo teve por objetivo analisar os gastos públicos em saúde destinados aos serviços ambulatoriais em Fonoaudiologia no Brasil a fim de demonstrar a importância do fortalecimento da prática da auditoria em saúde nesses serviços por profissionais fonoaudiólogos.

\section{MÉTODOS}

Trata-se de um estudo de caráter ecológico, com abordagem quantitativa, realizado por meio de busca em dados secundários disponíveis em plataforma virtual de domínio público, extraídos da base de dados do Sistema de Informações Ambulatoriais do Sistema Único de Saúde (SIA/SUS), vinculado ao Ministério da Saúde, fornecidos pelo Departamento de Informática do Sistema Único de Saúde (DATASUS), por meio do aplicativo TABNET (http://www.datasus.gov.br).

Foram adotados como critérios de inclusão todos os procedimentos das quatro grandes áreas da Fonoaudiologia (Audiologia, Linguagem, Motricidade Orofacial e Voz), além de dados financeiros em nível nacional entre os anos de 2009 e 2018 para os serviços fonoaudiológicos no âmbito do SUS, destinados à reabilitação (concessão de aparelhos auditivos), avaliação/diagnóstico e terapia. As etapas da coleta dos dados nos sistemas de informação no DATASUS estão detalhadas no fluxograma ilustrado na Figura 1.

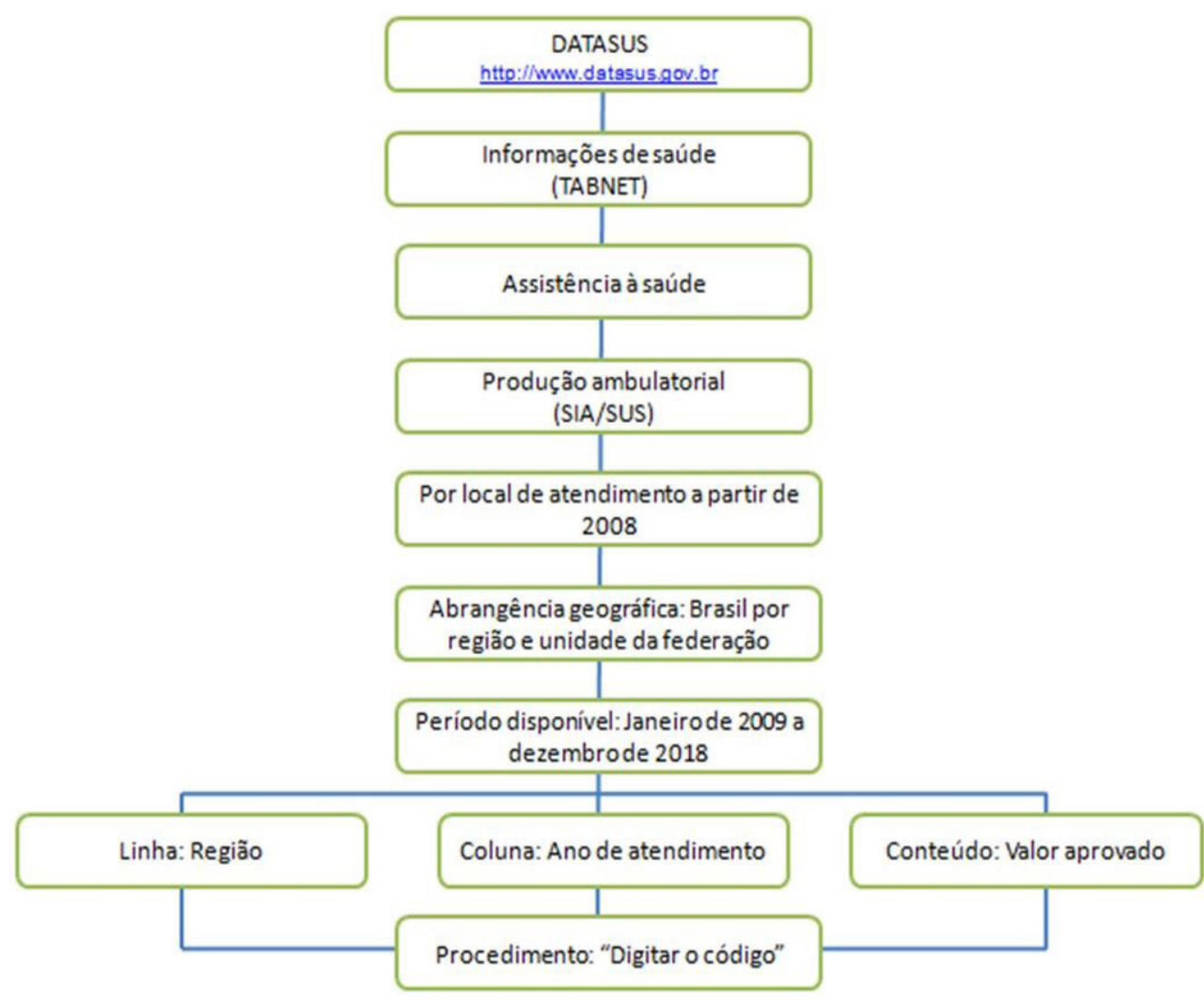

Figura 1. Fluxograma das etapas de coletas de dados na plataforma digital do DATASUS 
Não fizeram parte do presente estudo os códigos de procedimentos referentes ao implante coclear, próteses semi-implantáveis e/ou próteses ancoradas ao osso, próteses auriculares, condutas terapêuticas genéricas que inviabilizam a distinção da área de especialização a que se referem (Ex.: 0301070113 e 0301040044 ), bem como os procedimentos complementares voltados para terapia fonoaudiológica, uma vez que tais métodos não são exclusivos da Fonoaudiologia, sendo os recursos destinados para outras áreas.

No Quadro 1, estão catalogados os códigos dos procedimentos das quatro grandes áreas da Fonoaudiologia cadastrados no SIA/SUS. É válido expor que, até o momento, não há procedimentos cadastrados específicos à reabilitação para as áreas da Voz e Motricidade Orofacial no SIA/SUS, sendo o desta última referenciado por um código de cadastro genérico para o procedimento de avaliação.

A montagem do banco de dados foi realizada no programa computacional Microsoft Excel, versão 2010, sistema operacional Windows 10. Os recursos estatísticos adotados para a exposição dos dados foram as estatísticas descritivas, por meio de medida da taxa de crescimento, frequência relativa e absoluta. $\mathrm{O}$ método utilizado para calcular a taxa de crescimento anual foi por intermédio da diferença entre o ano anterior e o posterior, adotando o ano de 2009 como o marco zero.

O estudo, por apresentar caráter de análise de dados secundários, disponíveis em plataforma de domínio público, não foi submetido e registrado no sistema do CEP/CONEP, conforme a Resolução do Conselho Nacional de Saúde $\mathrm{n}^{\circ}$ 510/2016, porém, todos os princípios éticos envolvidos na análise dos dados foram cuidadosamente respeitados.

\section{RESULTADOS}

Por meio da análise dos dados referentes aos valores aprovados para os procedimentos ambulatoriais, a Tabela 1 apresenta a movimentação dos recursos públicos destinados ao financiamento da assistência com Fonoaudiologia, entre 2009 e 2018, nas Regiões brasileiras, destacando-se as quatro grandes áreas e os valores brutos (em reais) aprovados.

O montante investido nos dez anos analisados (2009-2018) somou um quantitativo de gastos de R $\$ 2.239 .526 .392,32 \mathrm{em}$ todo o Brasil. O investimento médio, por ano, foi de R\$ $223.952 .639,232$, sendo que $47,2 \%$ desse valor corresponderam exclusivamente aos recursos destinados à Região Sudeste $\mathrm{e}$ $5,7 \%$ para a Região Norte (Figura 2).

Evidencia-se que a área da Audiologia foi responsável por importante parcela $(95,4 \%)$ dos investimentos direcionados aos serviços em Fonoaudiologia nesse mesmo período (Tabela 1). No que tange às demais áreas voltadas para a terapia fonoaudiológica a maior parcela dos investimentos foi para a área da Linguagem ( $4,0 \%)$, seguida pelas áreas da Motricidade Orofacial $(0,5 \%)$ e da $\operatorname{Voz}(0,1 \%)$.

Pôde-se notar, ainda, que a área da Linguagem foi a responsável pelos maiores investimentos entre os procedimentos

Quadro 1. Relação dos códigos de procedimentos em Fonoaudiologia registrados no Sistema de Informação Ambulatorial do Sistema Único de Saúde incluídos no presente estudo

\begin{tabular}{|c|c|c|}
\hline Grande área & Procedimentos & Códigos de procedimentos \\
\hline \multirow[t]{2}{*}{ Audiologia } & Avaliação/Diagnóstico & $\begin{array}{l}0211070025,0211070033,0211070041,0211070122,0211070203,0211070211, \text {, } \\
0211070238,0211070262,0211070289,0211070327,0211070335,0211070343, \\
0211070050,0211070360,0211050113,0211070149,0211070157,0211070270, \\
0211070351,0211070092,0211070106,0211070297,0211070300 .\end{array}$ \\
\hline & Reabilitação & $\begin{array}{l}0211070319,0211070246,0301070032,0701030011,0701030020,0701030038 \text {, } \\
0701030046,0701030054,0701030062,0701030070,0701030089,0701030097, \\
0701030100,0701030119,0701030127,0701030135,0701030143,0701030151, \text {, } \\
0701030160,0701030178,0701030186,0701030194,0701030208,0701030216 \text {, } \\
0701030224,0701030232,0701030240,0701030259,0701030267,0701030275 \text {, } \\
0701030283,0701030291,0701030305 \text {. }\end{array}$ \\
\hline \multirow[t]{2}{*}{ Linguagem } & Avaliação & $0211070068,0211070076$. \\
\hline & Reabilitação & 0301070024. \\
\hline Voz & Avaliação & $0211070017,0211070114$. \\
\hline Motricidade orofacial & Avaliação & 0211070084. \\
\hline
\end{tabular}

Fonte: DATASUS - Sistema de Informação Ambulatorial do Sistema Único de Saúde - SIA/SUS (2009 a 2018)

Tabela 1. Valores aprovados em reais, destinados aos serviços fonoaudiológicos das diferentes Regiões da Federação Brasileira, nas quatro grandes áreas de atendimento (2009 a 2018)

\begin{tabular}{lrrrrr}
\hline \multirow{2}{*}{$\begin{array}{c}\text { Regiões da } \\
\text { federação }\end{array}$} & \multicolumn{2}{c}{ Valores aprovados por área de especialização da Fonoaudiologia (R\$) } & \multicolumn{2}{c}{ Audiologia } \\
\cline { 2 - 5 } & \multicolumn{1}{c}{$\begin{array}{c}\text { Motricidade } \\
\text { orofacial }\end{array}$} & Linguagem & \multicolumn{2}{c}{ Voz } & \multicolumn{2}{c}{ Total } \\
\hline Nordeste & $3.315 .610,98$ & $22.121 .627,87$ & $533.340,00$ & $487.031 .214,36$ & $513.001 .793,21$ \\
Norte & $1.109 .498,61$ & $2.636 .851,32$ & $134.851,00$ & $123.731 .167,20$ & $127.612 .368,13$ \\
Sudeste & $4.733 .326,71$ & $53.583 .270,57$ & $738.137,00$ & $997.417 .346,14$ & $1.056 .472 .080,42$ \\
Sul & $1.310 .746,13$ & $5.930 .350,28$ & $119.078,00$ & $355.705 .880,11$ & $363.066 .054,52$ \\
Centro-Oeste & $1.490 .797,01$ & $5.164 .936,52$ & $188.773,00$ & $172.529 .055,21$ & $179.373 .561,74$ \\
Brasil & $11.959 .979,44$ & $89.437 .570,86$ & $1.714 .179,00$ & $2.136 .414 .663,02$ & $2.239 .526 .392,32$ \\
\hline
\end{tabular}

Fonte: DATASUS - Sistema de Informação Ambulatorial do Sistema Único de Saúde - SIA/SUS (2009 a 2018)

Legenda: $\mathrm{R} \$=$ Reais 


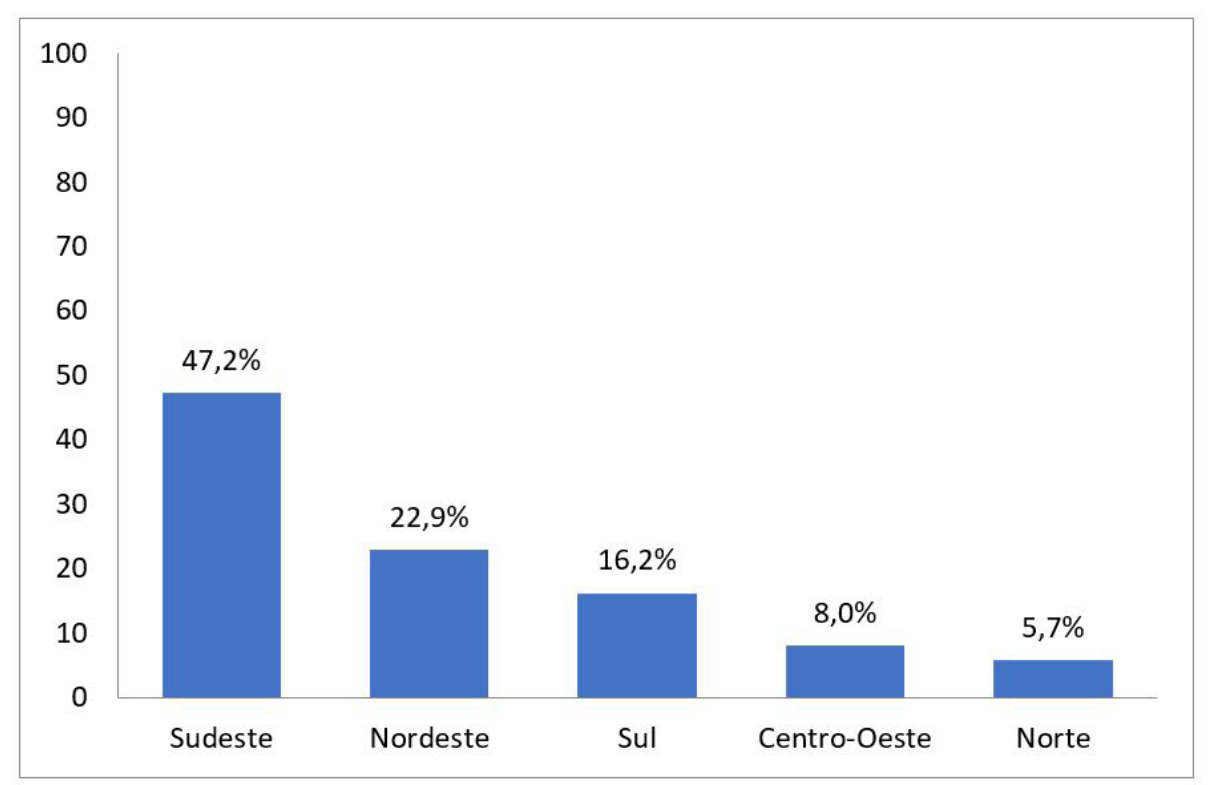

Figura 2. Distribuição dos custos ambulatoriais em Fonoaudiologia, por Região Federativa do Brasil, entre os anos de 2009 e 2018 . Fonte: DATASUS - Sistema de Informação Ambulatorial do Sistema Único de Saúde - SIA/SUS

Tabela 2. Distribuição dos valores aprovados e destinados aos atendimentos em Audiologia e respectivas taxas de crescimento anual entre os anos de 2009 e 2018

\begin{tabular}{|c|c|c|c|c|c|c|}
\hline \multirow[b]{2}{*}{ Período } & \multicolumn{5}{|c|}{ Recursos para os serviços de Audiologia (R\$) } & \multirow{2}{*}{$\begin{array}{c}\text { Tx } \\
\text { crescimento } \\
\text { anual (\%) }\end{array}$} \\
\hline & $\begin{array}{c}\text { Reabilitação } \\
\text { auditiva* }\end{array}$ & $\%$ & $\begin{array}{c}\text { Avaliação/Diagnóstico } \\
\text { audiológico }\end{array}$ & $\%$ & Total & \\
\hline 2009 & $80.314 .914,18$ & 49,4 & $82.207 .614,14$ & 50,6 & $162.522 .528,32$ & 0 \\
\hline 2010 & $93.510 .971,73$ & 50,6 & $91.245 .041,90$ & 49,4 & $184.756 .013,63$ & 12 \\
\hline 2011 & 103.623.149,46 & 51,0 & $99.945 .153,05$ & 49,0 & $203.568 .302,51$ & 9,2 \\
\hline 2012 & $107.213 .073,30$ & 51,2 & 102.180.496,89 & 49,8 & $209.393 .570,19$ & 2,8 \\
\hline 2013 & $112.172 .737,84$ & 51,9 & $103.980 .354,96$ & 49,1 & $216.153 .092,80$ & 3,1 \\
\hline 2014 & $119.217 .864,26$ & 52,7 & $106.900 .204,45$ & 47,3 & $226.118 .068,71$ & 4,4 \\
\hline 2015 & $116.919 .690,50$ & 51,8 & $108.632 .901,57$ & 48,2 & $225.552 .592,07$ & $-0,3$ \\
\hline 2016 & $124.479 .846,50$ & 52,9 & $110.679 .942,02$ & 47,1 & $235.159 .788,52$ & 4,1 \\
\hline 2017 & $119.481 .348,28$ & 51,4 & $112.897 .124,46$ & 48,6 & $232.378 .472,74$ & $-1,2$ \\
\hline 2018 & $124.713 .655,64$ & 51,8 & $116.094 .677,89$ & 48,2 & $240.808 .333,53$ & 3,5 \\
\hline
\end{tabular}

Fonte: Datasus - Sistema de Informação Ambulatorial do Sistema Unico de Saúde - SIA/SUS (2009 a 2018)

* Concessão, adaptação e avaliação de aparelhos auditivos de amplificação sonora

Legenda: $\mathrm{R} \$$ = Reais; $\mathrm{Tx}=$ Taxa; $\%$ = Percentual

de terapia fonoaudiológica em todas as Regiões Federativas, sendo as Regiões Sudeste $(5,1 \%)$ e Nordeste $(4,3 \%)$ as que apresentaram maiores parcelas dos recursos destinadas para esses setores, seguidas pelas Regiões Centro-Oeste $(2,9 \%)$, Norte $(2,1 \%)$ e Sul $(1,6 \%)$.

Para melhor expor os dados supracitados, a Tabela 2 divide a área da Audiologia em reabilitação auditiva e avaliação/diagnóstico audiológico, a fim de detalhar os gastos. A taxa de crescimento na área de Audiologia evidenciou um aumento gradativo dos custos nos procedimentos audiológicos, demonstrando crescimento nos primeiros anos, com posterior queda a partir do ano de 2011 e manutenção nos investimentos, com crescimento inferior, para os demais anos. Notou-se, também, que o segmento da reabilitação auditiva foi o responsável por mais da metade dos investimentos no âmbito da Audiologia, com porcentagens de distribuição dos recursos bem estabelecidas ao longo dos anos.

Do mesmo modo, ao analisar os valores aprovados para a assistência fonoaudiológica em terapia, entre os anos de 2009 e 2018, observou-se que as áreas de Motricidade Orofacial e Voz obtiveram decréscimos nas suas taxas de crescimento ao longo dos anos, sendo a área da Voz, quando comparada às demais áreas de especialização, a que apresentou investimentos inferiores. $\mathrm{Na}$ área da Linguagem, os valores se mantiveram praticamente estáveis e com pequenos acréscimos ao longo dos anos (Tabela 3).

No que tange à taxa de crescimento anual da assistência fonoaudiológica em terapia, verificou-se ligeira queda na taxa de crescimento dos valores aprovados a partir de 2010, com incremento importante no último ano, 2018. 
Tabela 3. Distribuição dos valores aprovados e destinados aos atendimentos em Motricidade Orofacial, Voz e Linguagem entre os anos de 2009 e 2018

\begin{tabular}{|c|c|c|c|c|c|c|c|c|}
\hline \multirow{2}{*}{ Período } & \multicolumn{6}{|c|}{ Investimento em serviços de fonoaudiológica (R\$) } & \multirow{2}{*}{ Total } & \multirow{2}{*}{$\begin{array}{c}\text { Tx } \\
\text { crescimento } \\
\text { anual (\%) }\end{array}$} \\
\hline & MO & $\%$ & Voz & $\%$ & Lgg & $\%$ & & \\
\hline 2009 & $1.144 .292,50$ & 13,4 & $178.474,00$ & 2,1 & $7.190 .551,72$ & 84,5 & $8.513 .318,22$ & 0 \\
\hline 2010 & $1.210 .748,46$ & 13,1 & $186.762,00$ & 2,0 & $7.841 .574,38$ & 84,9 & $9.239 .084,84$ & 7,9 \\
\hline 2011 & $1.150 .097,19$ & 12,6 & $197.687,00$ & 2,2 & $7.758 .905,62$ & 85,2 & $9.106 .689,81$ & $-1,5$ \\
\hline 2012 & $1.079 .955,93$ & 11,3 & $177.358,00$ & 1,9 & $8.290 .061,19$ & 86,8 & $9.547 .375,12$ & 4,6 \\
\hline 2013 & $1.097 .752,23$ & 10,8 & $187.778,00$ & 1,9 & $8.844 .040,49$ & 87,3 & $10.129 .570,72$ & 5,7 \\
\hline 2014 & $1.079 .105,16$ & 9,9 & $177.894,00$ & 1,6 & $9.655 .973,50$ & 88,5 & $10.912 .972,66$ & 7,2 \\
\hline 2015 & $1.187 .263,92$ & 11,6 & $170.248,00$ & 1,7 & $8.918 .473,48$ & 86,8 & $10.275 .985,40$ & $-6,2$ \\
\hline 2016 & $1.279 .595,07$ & 11,9 & $156.324,00$ & 1,5 & $9.252 .231,26$ & 86,6 & $10.688 .150,33$ & 3,8 \\
\hline 2017 & $1.300 .079,31$ & 11,7 & $142.781,00$ & 1,3 & $9.628 .042,32$ & 87,0 & $11.070 .902,63$ & 3,5 \\
\hline 2018 & $1.431 .089,67$ & 10,5 & $138.873,00$ & 1,0 & $12.057 .716,90$ & 88,5 & $13.627 .679,57$ & 18,8 \\
\hline
\end{tabular}

Fonte: DATASUS - Sistema de Informação Ambulatorial do Sistema Único de Saúde - SIA/SUS (2009 a 2018)

Legenda: $\mathrm{MO}=$ Motricidade Orofacial; Lgg = Linguagem; Tx = Taxa; R\$ = Reais; \% = Percentual

\section{DISCUSSÃO}

Pôde-se observar que a média das despesas públicas anuais com Fonoaudiologia foi de, aproximadamente, R\$223.952.639,232; distribuídos na assistência em diversas especialidades. É válido ressaltar que o valor supracitado pode estar subnotificado e desatualizado e, eventualmente, superior ao demonstrado no presente estudo.

Esses achados atestam que a Fonoaudiologia é responsável por uma parcela importante dos gastos em procedimentos ambulatoriais, tanto em média, quanto em alta complexidade, em todas as Regiões da Federação, com indicadores de crescimento nos atendimentos e custos ao longo dos anos, diretamente relacionados à demanda da produção ambulatorial e às novas políticas públicas de saúde.

Esses procedimentos vêm sendo implantados no decorrer dos últimos anos e passaram a ser garantidos mediante programas e leis, como a Política Nacional de Atenção à Saúde Auditiva (GM/ MS 2073/04) ${ }^{(4)}$, Teste da Orelhinha (Lei Federal n $\left.{ }^{0} 12.303 / 2010\right)^{(5)}$, Portaria $n^{\circ} 793 / 2012$, que institui a Rede de Cuidados à Pessoa com Deficiência no Âmbito do SUS ${ }^{(6)}$, Protocolo de Avaliação do Frênulo da Língua em Bebês (Lei n $\left.{ }^{\circ} 13.002 / 2014\right)^{(7)}$, atualmente sistematizados na Portaria de Consolidação n 3/GM/MS/2017, que consolida as normas sobre a Rede de Cuidados à Pessoa com Deficiência no Sistema único de Saúde ${ }^{(8)}$, dentre outras políticas que preveem a participação de fonoaudiólogos nas equipes de diferentes serviços de atenção à saúde.

Nesse sentido, os serviços de Fonoaudiologia no SUS vêm crescendo com o passar dos anos ${ }^{(1)}$. Nota-se que, no Brasil, atualmente há 22.398 (vinte e dois mil, trezentos e noventa e oito) serviços de Fonoaudiologia cadastrados no Cadastro Nacional de Estabelecimentos de Saúde (CNES) ${ }^{(9)}$ e milhões de usuários em tratamento em nível ambulatorial e hospitalar em Fonoaudiologia, anualmente. A Fonoaudiologia é uma área de atuação profissional que, ao longo dos anos, tem mostrado o seu protagonismo nas ações de saúde no âmbito do SUS, desde a atenção básica até os níveis de alta complexidade ${ }^{(10)}$.

Os aspectos da audição, linguagem, fala e voz, objetos de estudo da Fonoaudiologia, são considerados atributos da saúde global, já que alterações nesses sistemas têm potenciais para afetar não somente as competências comunicativas verbais e não verbais, mas gerar limitações na capacidade de o indivíduo explorar o mundo, ocasionando impactos na experiência pessoal e social ${ }^{(11)}$. Assim, a Fonoaudiologia tem demonstrado a sua importância nos serviços de saúde, provando ser cada vez mais necessária no âmbito das equipes multidisciplinares em todos os níveis de complexidade.

Conforme os achados deste estudo, notou-se a presença de assimetria na distribuição dos montantes referentes aos gastos para os serviços fonoaudiológicos entre as diferentes Regiões Federativas, sendo a Região Sudeste a que retém a maior parcela dos recursos públicos, seguida pela Região Nordeste. O menor montante de custos para os mesmos serviços foi destinado para a Região Norte.

No Brasil, de acordo com o último Censo do Instituto Brasileiro de Geografia e Estatística (IBGE) do ano de 2010 ${ }^{(12)}$, as Regiões Sudeste e Nordeste demonstraram maior número de habitantes por metros quadrados, o que poderia justificar, em parte, os altos investimentos pela demanda ${ }^{(13)}$ e um maior quantitativo de serviços de Fonoaudiologia cadastrados no CNES e de profissionais fonoaudiólogos na região. Já a Região Norte apresentou o menor quantitativo de habitantes por metro quadrado.

Essa modalidade de distribuição dos serviços de saúde em Fonoaudiologia pode ser reflexo da normatização estabelecida por programas de saúde anteriores, em especial a saúde auditiva, que representa a maior parcela dos gastos e por muitos anos seguiu os parâmetros da Portaria SAS/MS no 587/04, que preconizou o quantitativo de um serviço de saúde auditiva a cada estado com população inferior a 1,5 milhões de habitantes e dois serviços aos estados com população entre 2 e 3 milhões de habitantes ${ }^{(14)}$.

No presente estudo ecológico, evidenciou-se que os serviços destinados à assistência audiológica foram responsáveis pela maior parcela dos investimentos em Fonoaudiologia em todas as Regiões Federativas, quando comparados aos serviços de terapia fonoaudiológica, em que as áreas da Motricidade Orofacial e Voz, em respectivo, obtiveram os menores custos registrados.

Essa distribuição desproporcional entre os diferentes tipos de assistência fonoaudiológica está relacionada ao fato de a área da Audiologia incluir um portfólio de serviços e procedimentos de alto custo que envolvem, além das avaliações comportamentais, eletroacústicas e eletrofisiológicas, a reabilitação auditiva por meio de concessões de aparelhos de amplificação sonora individual (AASI) de diferentes tecnologias, bem como os 
procedimentos terapêuticos destinados às diversas patologias do sistema auditivo e vestibular, os quais estão distribuídos em todos os níveis de complexidade de saúde.

Cita-se, ainda, o fato de que a Audiologia, por muitos anos, teve os seus serviços e procedimentos assegurados pela Política Nacional de Atenção à Saúde Auditiva (PNASA) que garantiu o diagnóstico e reabilitação auditiva nas redes de saúde especializadas do SUS. Notou-se, também, uma variedade de procedimentos destinados à saúde auditiva com cadastro ativo na base de dados do SIA/SUS, o que não ocorre nas áreas destinadas à terapia, situação que pode contribuir, em parte, para essa discrepância.

Constatou-se, entretanto, que poucos procedimentos relacionados à assistência terapêutica estão cadastrados na base de dados do DATASUS, especialmente aqueles das áreas da Motricidade Orofacial e Voz, e que muitos deles apresentam uma definição geral, frequentemente insuficiente para caracterizar procedimentos específicos na área. No entanto, embora os cadastros no SIA/ SUS sirvam de termômetro socioeconômico e financeiro podem também conter registros incorretos nessa plataforma de domínio público, o que justificaria as subnotificações e os baixos valores aplicados a esses procedimentos. Nesse sentido, salienta-se a importância da criação de novos códigos destinados a cada área e específicos para cada procedimento.

Observou-se, ainda, que as taxas de crescimento tiveram uma queda a partir do ano de 2011, decréscimo compatível com o período em as políticas públicas de saúde auditiva passaram por uma reformulação, ocasionando o fim do PNASA que abrangia desde ações de promoção da saúde auditiva até a terapia fonoaudiológica em todos os ciclos da vida ${ }^{(15)}$.

Apesar de os dados apontarem que os procedimentos fonoaudiológicos consomem parcela importante dos recursos públicos, a área da assistência audiológica carece de maior atenção em termos de especificidade na auditoria, considerando que estes são auditados por outros profissionais da área da saúde sem os domínios de conhecimentos técnicos para os procedimentos específicos da área, o que compromete a qualidade da auditoria na Fonoaudiologia e, por conseguinte, na assistência em saúde prestada aos usuários do SUS.

É válido apontar que a auditoria em saúde serve como forma de garantir o acesso do usuário aos serviços ambulatoriais em Fonoaudiologia de forma efetiva e consciente, proporcionando um crescimento de forma organizada, menos oneroso, eficaz e de qualidade nos sistemas de saúde.

Somado a isso, o Sistema Nacional de Auditoria (SNA) não dispõe, ainda, de protocolos específicos que possam sistematizar a atividade em auditoria na área de Fonoaudiologia, como acontecem de forma bem consolidada na Medicina, Enfermagem e Odontologia, áreas que dispõem de protocolos específicos junto ao SNA. Tal situação pode explicar a fragilidade da auditoria nos serviços de saúde, em especial na assistência fonoaudiológica, fato que está em desacordo com o preconizado pelo SNA, o qual defende que o exercício da auditoria no SUS deve ser desenvolvido por uma equipe multidisciplinar a fim de aumentar a eficiência e a resolutividade da função por meio da integração dos profissionais envolvidos nos mais variados serviços integrados à rotina do sistema ${ }^{(16)}$.

Relatos na literatura demonstram dificuldades dos auditores de saúde para auditar serviços que não constituem sua área do conhecimento, já que o exercício da auditagem em serviços distintos da área de formação primária do auditor exige muito do conhecimento técnico específico daquele serviço auditado, além da experiência dos auditores sobre os procedimentos, funcionamento dos serviços e equipamentos. Não raro, verificase em estudos relatos de auditores que apontam a carência de outros profissionais nas equipes ${ }^{(17)}$.

Assim, a auditoria, como ferramenta de qualidade eficiente que aprimora um sistema de gestão, é essencial na estrutura regimental dos serviços privados e públicos. Quando bem aplicada, diagnosticará não-conformidades no sistema avaliado e determinará uma forma de acrescentar melhorias às políticas públicas em curso, a partir da otimização dos recursos para o atendimento de qualidade ${ }^{(18)}$

A inserção da avaliação e controle sistemático, como forma de organização nos serviços de saúde, é observada como um recurso válido, tanto para usuários, quanto para os profissionais e gestores, além do fornecimento de informações contribuírem para o foco na observação de programas, serviços ou tecnologias ${ }^{(19,20)}$.

Os dados deste estudo, portanto, levam à reflexão de que é importante e urgente a existência de um controle rigoroso e mais específico dos recursos públicos destinados aos serviços de Fonoaudiologia, desde que exercido por um profissional desta categoria, com vistas a verificar, de forma mais criteriosa, os fatores que agem nessa demanda, bem como as estratégias de gestão que possam viabilizar maior qualidade na prestação desse serviço à população, conforme regulamentado pelo Conselho Federal de Fonoaudiologia que criou a Resolução CFFa n ${ }^{\circ} 455$, de 30 de outubro de $2014^{(21)}$.

Dada essa realidade, somada à significativa escassez de publicações sobre esse tema na Fonoaudiologia, o presente estudo debateu-se com diversas limitações, a fim de comprovar os dados apresentados de forma mais contundente. Além disso, tais dados podem ter sido mal representados em virtude das subnotificações na plataforma do SIA/SUS, o que comprometeu a real análise dos valores, visto que, alguns procedimentos estavam incompletos, faltando registro de algumas regiões, bem como valores registrados não condizentes com os procedimentos. Contudo, os registros na plataforma do SIA/SUS não podem ser desvalorizados, pois servem para demonstrar o quantitativo de procedimentos que a Fonoaudiologia tem realizado na assistência à saúde, em especial no âmbito do SUS.

Nesse sentido, faz-se necessário, também, que o Conselho Federal de Fonoaudiologia e os Conselhos Regionais discutam e repensem a importância dessa prática, busquem articular junto aos gestores de saúde a criação de protocolos específicos na auditoria em Fonoaudiologia, assim como analisar a necessidade da criação de uma nova área de especialização fonoaudiológica voltada para a auditoria em saúde a fim de que os fonoaudiólogos possam conhecer esse novo campo, se especializar e auxiliar na otimização dos recursos, minimizando desperdícios e, assim, gerar maior qualidade na prestação dos serviços para os usuários.

\section{CONCLUSÃO}

Os gastos públicos em saúde destinados aos serviços de assistência em Fonoaudiologia apresentam um montante considerável, sendo a área assistencial da Audiologia a responsável por mais de $90 \%$ dos investimentos nos serviços públicos. Esses gastos encontram-se distribuídos de maneira desigual entre as diferentes Regiões do Brasil, ao se considerar que quase metade desse valor corresponde à Região Sudeste e uma pequena parcela, à Região Norte. 
Ficou evidente a importância de que os serviços fonoaudiológicos sejam auditados por profissionais com qualificação técnica específica na área a fim de oferecer uma revisão dos gastos de forma mais criteriosa, uma vez que os gastos aplicados aos serviços fonoaudiológicos têm apresentado um padrão de crescimento em ritmo acelerado, estando boa parte dos recursos destinados a todos os serviços nos diferentes níveis de complexidade, nos quais o atendimento à assistência ambulatorial se destaca como o principal responsável por esse crescimento dos gastos.

\section{REFERÊNCIAS}

1. MoreiraMD, MotaHB. Os caminhos da fonoaudiologia no Sistema Único de Saúde - SUS.Rev CEFAC. 2009;11(3):516-21. http://dx.doi. org/10.1590/S1516-18462009000300021.

2. ViacavaF, UgáMAD, PortoS, LaguardiaJ, MoreiraRS. Avaliação de Desempenho de Sistemas de Saúde: um modelo de análise.Cien Saude Colet. 2012;17(4):921-34. http://dx.doi.org/10.1590/S141381232012000400014. PMid:22534846.

3. Brasil. Ministério da Saúde. Financiamento público de saúde / Ministério da Saúde, Organização Pan-Americana da Saúde. Ministério da Saúde; Brasília; 2013.

4. Brasil. Ministério da Saúde. Portaria ${ }^{\circ} 2073$, de 28 de setembro de 2004. Institui a política nacional de atenção à saúde auditiva. Diário Oficial da União [Internet]; Brasília; 2004 [citado em 2020 Abr 06]. Disponível em: http://bvsms.saude.gov.br/bvs/saudelegis/gm/2004/ prt2073_28_09_2004.html.

5. Brasil. Lei Federal $n^{0} 12.303 / 10$, de 2 de agosto de 2010. Dispõe sobre a obrigatoriedade de realização do exame denominado Emissões Otoacústicas Evocadas. Diário Oficial da União [Internet]; Brasília; 2010 [citado em 2020 Abr 06]. Disponível em: http://www.planalto. gov.br/ccivil_03/_Ato2007-2010/2010/Lei/L12303.htm.

6. Brasil. Ministério da Saúde. Portaria no 793, de 24 de abril de 2012. Institui a rede de cuidados à pessoa com deficiência no âmbito do Sistema Único de Saúde. Diário Oficial da União [Internet]; Brasília; 2012 [citado em 2020 Mar 05]. Disponível em: http://bvsms.saude. gov.br/bvs/saudelegis/gm/2012/prt0793_24_04_2012.html.

7. Brasil. Cartilha do teste da linguinha: para mamar, falar e viver melhor. Pulso Editorial [Internet]; São José dos Campos; 2014 [citado em 2021 Mar 31]. Disponível em: https://www.sbfa.org.br/fono2014/ pdf/testelinguinha_2014_livro.pdf.

8. Brasil. Ministério da Saúde. Portaria de consolidação $\mathrm{n}^{\circ} 3$ de setembro de 2017. Consolidação das normas sobre as redes do Sistema Único de Saúde. Diário Oficial da União [Internet]; Brasília; 2017 [citado em 2021 Mar 31]. Disponível em: http://bvsms.saude.gov.br/bvs/ saudelegis/gm/2017/prc0003_03_10_2017.html.

9. Brasil. Secretaria de Atenção à Saúde. Cadastro Nacional de Estabelecimentos de Saúde: CNESNet. CNESNet [Internet]; Brasília; 2020 [citado em 2020 Abr 01]. Disponível em: http://cnes2.datasus. gov.br/Mod_Ind_Profissional_com_CBO.asp.

10. Conselho Federal de Fonoaudiologia. Contribuição da Fonoaudiologia para o avanço do SUS - 27 anos do SUS: a Fonoaudiologia na conquista pela integralidade da atenção à saúde. Brasília: Sistema de Conselhos de Fonoaudiologia; 2015. 24 p.

11. AndradeCRF. Fases e níveis de prevenção em fonoaudiologia: ações coletivas e individuais. In: VieiraRM,organizador. Fonoaudiologia e saúde coletiva. 2. ed. Carapicuíba: Pró-Fono; 2000. 104 p.

12. Brasil. Ministério do Planejamento, Orçamento e Gestão. Instituto Brasileiro de Geografia e Estatística - IBGE. Censo Demográfico 2010. Características gerais da população, religião e pessoas com deficiência. IBGE; Rio de Janeiro; 2013.

13. AndradeCL, FernandesL, RamosHE, MendesCMC, AlvesCAD. Programa Nacional de Atenção à Saúde Auditiva: avanços e entraves da saúde auditiva no Brasil.Rev Ciênc Méd Biol. 2013;12(1):404-10.

14. Brasil. Secretaria de Atenção à Saúde. Portaria $n^{\circ} 587$, de 07 de outubro de 2004. Diário Oficial da União [Internet]; Brasília; 2004 [citado em 2020 Mai 07]. Disponível em: http://bvsms.saude.gov.br/ bvs/saudelegis/sas/2004/prt0587_07_10_2004.html.

15. SilvaLSG, GonçalvesCGO, SoaresVMN. Política Nacional de Atenção à Saúde Auditiva: um estudo avaliativo a partir da cobertura de serviços e procedimentos diagnósticos. CoDAS. 2014;26(3):241-7.

16. Brasil. Ministério da Saúde. Departamento Nacional de Auditoria do SUS. Auditoria no SUS: noções básicas sobre sistemas de informação. Ministério da Saúde; Brasília; 2004.

17. AleluiaÍRS, SantosFC. Auditoria em fisioterapia no Sistema Único de Saúde: proposta de um protocolo específico. Fisioter Mov. 2013;26(4):725-41.

18. SantosLC, BarcellosVF. Auditoria em saúde: uma ferramenta de gestão. Monografia (Pós-Graduação em Gestão e Auditoria em Saúde). Brasília: Centro Universitário UNIEURO; 2009.

19. BevilacquaMC, MeloTM, MorettinM, LopesAC. A avaliação de serviços em Audiologia: concepções e perspectivas. Rev Soc Bras Fonoaudiol. 2009;14(3):421-6.

20. SilvaKR, FerreiraMC, GuiaACOM, OliveiraNR, LemosSMA. Produção científica em saúde auditiva no Brasil: análise do período de 2000 a 2010. Rev CEFAC. 2013;15(1):215-27.

21. Brasil. Conselho Federal de Fonoaudiologia. Resolução CFFa n ${ }^{\circ} 455$ de 30 de outubro de 2014. Dispõe sobre a auditoria em Fonoaudiologia e dá outras providências. Diário Oficial da União; Brasília; 2014. 